Journal of Engineering and Applied Sciences 14 (22): 8348-8355, 2019

ISSN: 1816-949X

(C) Medwell Journals, 2019

\title{
Stochastic Neutron Density Employing Different Uncertainties
}

\author{
${ }^{1}$ Daniel Suescun-Diaz, ${ }^{2}$ Jorge H. Figueroa-Jimenez and ${ }^{1}$ Yohan M. Oviedo-Torres \\ ${ }^{1}$ Departamento de Ciencias Naturales, Facultad de Ciencias Exactas y Naturales, \\ Grupo de Fisica Aplicada FIASUR, Universidad Surcolombiana, \\ Av. Pastrana Borrero-Carrera 1, CP 410006 Neiva, Colombia \\ ${ }^{2}$ Departamento de Ciencias Naturales y Matematicas, Facultad de Ingenieria y Ciencias, \\ Grupo de Estadistica y Matematica Aplicada, Pontificia Universidad Javeriana Cali, \\ Calle 18 No. 118-250, Cali, Colombia
}

\begin{abstract}
In this study, a numerical study of the stochastic dynamics of a nuclear reactor is carried out using the Euler-Maruyama method to resolve the stochastic point kinetic equations. The method was tested using different uncertainties, initial conditions and different forms of reactivity with one and six groups of delayed neutron precursors using up to 5000 Brownian trajectories for each numerical results. The average expected values, standard deviations of neutron density and delayed neutron precursors were calculated in each computational experiment. It was compared with other methods reported in literature and on average with the deterministic model of point kinetics, demonstrating that the Euler-Maruyama method for this proposal, apart from being easy to implement is extremely precise in obtaining expected values.
\end{abstract}

Key words: Brownian motion, Euler-Maruyama, neutron density, nuclear reactors, stochastic point kinetic equations, literature

\section{INTRODUCTION}

A nuclear reactor is a device in which controlled nuclear reactions are carried out to free energy. The dynamics of this device is described in deterministic form by point kinetic equations which are a set of strongly linked non-linear differential equations which describe an average value of the neutron density and delayed neutron precursor concentrations. However, in levels of low power, high randomness occurs in the dynamics of the neutrons in the presence of a weak source (Stacey, 2007), therefore, the process must be described with the stochastic point kinetic equations.

There are various researches in literature in which the stochastic point kinetic equations are solved: the Piecewise Constant Approximation (PCA) and Monte Carlo (Hayes and Allen, 2005), Euler-Maruyama and Taylor 1.5 (Ray, 2012; Ray and Patra, 2013) the Simplified Stochastic Point Kinetics (SSPK) (Ayyoubzadeh and Vosoughi, 2014) the Analytical Exponential Model (AEM) (Nahla and Edress, 2016a) the Efficient Stochastic Model (ESM) (Nahla and Edress, 2016b) and the Double Diagonalization-Decomposition Method (DDDM) (Da Silva et al., 2016).

In this research, due to the difficulty in generating sufficiently random numbers, consideration was given to different seeds, this is a set of values to begin the generation of random numbers by means of the randn function of MATLAB and with this, simulation can be carried out of different Brownian motions whose differential is known as white noise which express as uncertainties which serve to resolve the stochastic point kinetic equation using the Euler-Maruyama method. Considering the average of numerical experiments obtained for each seed, the results are compared with the methods reported in literature and on average with the deterministic model calculated with Runge-Kutta $\mathrm{O}\left(\mathrm{h}^{4}\right)$.

\section{MATERIALS AND METHODS}

The stochastic point kinetic equations: It is possible to obtain the stochastic point kinetic equations based on the analysis of the events of birth and capture of neutrons and to assume that the changes in neutron population follow a normal distribution (Hayes and Allen, 2005). This analysis results from the following non-linear system of $\mathrm{m}+1$ Ito stochastic differential equations for the neutron density and delayed neutron precursor concentrations:

$$
\frac{d \vec{P}(t)}{d t}=A \vec{P}(t)+\vec{Q}(t)+B^{\frac{1}{2}} \frac{d \vec{W}(t)}{d t}
$$

Corresponding Author: Daniel Suescun-Diaz, Universidad Surcolombiana, CP 410006 Neiva, Colombia, daniel.suescun@usco.edu.co,(57) (8) 8754753 Ext. 1069 
Where:

$$
\vec{P}(t)=\left[\begin{array}{c}
n(t) \\
C_{1}(t) \\
C_{2}(t) \\
\vdots \\
C_{m}(t)
\end{array}\right] \vec{Q}(t)=\left[\begin{array}{c}
q(t) \\
0 \\
0 \\
\vdots \\
0
\end{array}\right] \vec{W}(t)=\left[\begin{array}{c}
\mathrm{W}_{1}(t) \\
W_{2}(t) \\
W_{3}(t) \\
\vdots \\
W_{m+1}(t)
\end{array}\right]
$$

for $\mathrm{i}=1, \ldots, \mathrm{m}$, where, $\mathrm{m}$ is the number of groups of concentration of delayed neutrons precursors $n(t)$ and $\mathrm{C}_{\mathrm{i}}(\mathrm{t})$ are the neutron density and the ith density of delayed neutrons precursors, respectively, $q(t)$ is the external source of neutrons and $\mathrm{W}_{1}(\mathrm{t}), \mathrm{W}_{2}(\mathrm{t}), \ldots, \mathrm{W}_{\mathrm{m}+1}(\mathrm{t})$ are Wiener processes or Brownian motion and $\mathrm{dW}_{1}(\mathrm{t})$, $\mathrm{dW}_{2}(\mathrm{t}), \ldots, \mathrm{dW}_{\mathrm{m}+1}(\mathrm{t})$ are uncertainties or white noise. On the other hand, the matrices $\mathrm{A}$ and $\mathrm{B}$ are given by:

$$
\begin{aligned}
\mathrm{A} & =\left[\begin{array}{ccccc}
\frac{\rho(\mathrm{t})-\beta}{\Lambda} & \lambda_{1} & \lambda_{2} & \cdots & \lambda_{\mathrm{m}} \\
\frac{\beta_{1}}{\Lambda} & -\lambda_{1} & 0 & \cdots & 0 \\
\frac{\beta_{2}}{\Lambda} & 0 & -\lambda_{2} & \cdots & 0 \\
\vdots & \vdots & \vdots & \ddots & \vdots \\
\frac{\beta_{\mathrm{m}}}{\Lambda} & 0 & 0 & \cdots & -\lambda_{\mathrm{m}}
\end{array}\right] \\
\mathrm{B} & =\left[\begin{array}{ccccc}
\zeta & \mathrm{a}_{1} & \mathrm{a}_{2} & \cdots & \mathrm{a}_{\mathrm{m}} \\
\mathrm{a}_{1} & \mathrm{~b}_{1,1} & \mathrm{~b}_{1,2} & \cdots & \mathrm{b}_{1, \mathrm{~m}} \\
\mathrm{a}_{2} & \mathrm{~b}_{2,1} & \mathrm{~b}_{2,2} & \cdots & \mathrm{b}_{2, \mathrm{~m}} \\
\vdots & \vdots & \vdots & \ddots & \vdots \\
\mathrm{a}_{\mathrm{m}} & \mathrm{b}_{\mathrm{m}, 1} & \mathrm{~b}_{\mathrm{m}, 2} & \cdots & \mathrm{b}_{\mathrm{m}, \mathrm{m}}
\end{array}\right]
\end{aligned}
$$

Doing the required multiplication, using Eq. $4 \mathrm{a}$ and 2 in the term $\mathrm{B}^{1 / 2} \mathrm{dW}(\mathrm{t})$ given in Eq. 1, provides that to each variable contained in $\vec{P}(t)$ would give us $(m+1)$ uncertainties, this is one for neutrons density and $\mathrm{m}$ for each concentration of precursors as can be seen in Eq. 1-2.

B represents the covariance matrix as it is in Eq. 4a, it is a symmetric, positive matrix where the main diagonal represents the variance and the other elements represent the covariance. With the disadvantage of calculating $\mathrm{B}^{1 / 2}$ that would give a computational effort. We propose that the calculation of each of the variables contained in $\overrightarrow{\mathrm{P}}(\mathrm{t})$ is associated with only one uncertainty and that it depends on the same variable that will be calculated, this means that we assume that the variables contained in $\overrightarrow{\mathrm{P}}(\mathrm{t})$ have zero covariance, this it is possible to write the Eq. 4a in the following way:

$$
\mathrm{B}^{* *}=\left[\begin{array}{ccccc}
\zeta & 0 & 0 & \cdots & 0 \\
0 & \mathrm{~b}_{1,1} & 0 & \cdots & 0 \\
0 & 0 & \mathrm{~b}_{2,2} & \cdots & 0 \\
\vdots & \vdots & \vdots & \ddots & \vdots \\
0 & 0 & 0 & \cdots & \mathrm{b}_{\mathrm{m}, \mathrm{m}}
\end{array}\right]
$$

where, the elements of matrix $\mathrm{B}$ or $\mathrm{B}^{* *}$ are defined as:

$$
\begin{gathered}
\zeta=\left[\frac{-1-\rho(t)+2 \beta+(1-\beta)^{2} v}{\Lambda}\right] n(t)+\sum_{i=1}^{m} \lambda_{i} C_{i}(t)+q(t) \\
a_{i}=\frac{\beta_{1}}{\Lambda}[(1-\beta) v-1] n(t)-\lambda_{i} C_{i}(t) \\
b_{i, j}=\frac{\beta_{i} \beta_{j} v}{\Lambda} n(t)+\delta_{i, j} \lambda_{i} C_{i}(t)
\end{gathered}
$$

Where:

$\mathrm{p}(\mathrm{t}) \quad=$ The reactivity

$v \quad=$ The average neutron number per fission

$\lambda_{\mathrm{i}}=$ The decay constant of the ith precursor which emits delayed neutron

$\beta_{\mathrm{i}}=$ The fraction of delayed neutrons of the ith group of precursors

$\beta=\sum_{\mathrm{i}=1}^{\mathrm{m}} \beta_{\mathrm{i}}=$ The total fraction of delayed neutrons

$\Lambda \quad=$ The prompt neutron generation time

$\delta_{i, j}=$ The Kronecker delta, defined in accordance with the following Eq. 9

$$
\delta_{1, j}=\left\{\begin{array}{l}
1 \text { if } i=j \\
0 \text { if } i \neq j
\end{array}\right.
$$

Equation 1 is known as the stochastic point kinetic equations and generalizes the deterministic equations, since when $\mathrm{B}=0$, it returns to the deterministic model (Kinard and Allen, 2004). The importance of $\left(\mathrm{B}^{* *}\right)$ is that it is not required to compute the square root of a matrix which is a great advantage when compared with $B$. The matrix $\mathrm{B}^{* *}$ was found in the literature (Ayyoubzadeh and Vosoughi, 2014) considering a poisson distribution, using the stirling's approximation, stable distributions with the disadvantage of additionally requiring two Wiener processes to solve Eq. 1 and finally using the independence of normally distributed random variables to simplify the problem. In this research, it was necessary to suppose only the covariance is zero.

The Euler-Maruyama method: The Euler-Maruyama approximation is a continuous multidimensional stochastic process $\overrightarrow{\mathrm{Y}}_{\mathrm{t}}=\left\{\overrightarrow{\mathrm{Y}}(\mathrm{t}), \mathrm{t}_{0}<\mathrm{t}<\mathrm{T}\right\}$ which satisfies the following iterative scheme: 


$$
\overrightarrow{\mathrm{Y}}_{\mathrm{n}+1}=\overrightarrow{\mathrm{Y}}_{\mathrm{n}}+\mathrm{a}\left(\mathrm{t}_{\mathrm{n}}, \overrightarrow{\mathrm{Y}}_{\mathrm{n}}\right) \mathrm{h}+\mathrm{b}\left(\mathrm{t}_{\mathrm{n}}, \overrightarrow{\mathrm{Y}}_{\mathrm{n}}\right) \Delta \overrightarrow{\mathrm{W}}
$$

For $\mathrm{n}=0,1,2, \ldots, \mathrm{N}-1$ where $\overrightarrow{\mathrm{Y}}_{0}=\overrightarrow{\mathrm{Y}}\left(\mathrm{t}_{0}\right)=\left[\mathrm{Y}_{0}^{1}, \mathrm{Y}_{0}^{2}, \mathrm{Y}_{0, \ldots}^{3}, \mathrm{Y}_{0}^{\mathrm{k}}\right]^{\mathrm{T}}$ is defined as the initial conditions, the time step defined as $\mathrm{h}=\mathrm{t}_{\mathrm{n}+1}-\mathrm{t}_{\mathrm{n}}$ and $\Delta \overrightarrow{\mathrm{w}}=\overrightarrow{\mathrm{w}}_{\mathrm{n}+1}-\overrightarrow{\mathrm{w}}_{\mathrm{n}}$ is the increase of the Brownian motion or uncertainties defined as $\Delta \overrightarrow{\mathrm{W}}=\sqrt{\mathrm{h} \vec{\eta}}$ where, $\eta$ is a vector of random variables normally distributed with a mean zero and unit variance. Applying the Euler-Maruyama method given for Eq. 10 to the expression given for Eq. 1 gives the result:

$$
\bar{P}_{n+1}=\vec{P}_{n}+\left(A_{n} \vec{P}_{n}+\vec{Q}_{n}\right) h+B_{\frac{n}{2}}^{\frac{1}{2}} \Delta \vec{W}
$$

With initial condition $\overrightarrow{\mathrm{P}}_{0}=\overrightarrow{\mathrm{Y}}_{0}$. Equation 11 is the solution of the point kinetic stochastic equations using the Euler-Maruyama method in Eq. 1.

\section{RESULTS AND DISCUSSION}

The following are the results of the proposed method, using different uncertainties $\Delta \overrightarrow{\mathrm{W}}$ for multiple computational experiments. Calculation of the expected values and standard deviations for different uncertainties is carried out by means of different seeds. The results are subsequently compared with stochastic methods reported in literature such as PCA and Monte Carlo (Hayes and Allen, 2005), Euler-Maruyama and Taylor 1.5 (Saha, 2012, 2013), AEM (Nahla and Edress, 2016a) ESM (Nahla and Edress, 2016b) DDDM (Da Silva et al., 2016) and on average with Runge-Kutta $\mathrm{O}\left(\mathrm{h}^{4}\right)$ deterministic. In all the computational experiments shown, up to 5000 Brownian trajectories were used for each seed.

To test the proposed method, the first experiment consists of a step-reactivity insertion $\rho=-1 / 3$ when one precursor $(m=1)$ is considered in a nuclear reactor with the following parameters: decay constant $\lambda_{1}=0.1 \mathrm{sec}^{-1}$, fraction of delayed neutrons $\beta_{1}=0.05$, average neutron number per fission $\nu=2.5$, generation time $\Lambda=2 / 3 \mathrm{sec}$, external source $\mathrm{q}=200 \mathrm{sec}^{-1}$ and an initial condition of $\overrightarrow{\mathrm{P}}_{0}=[400,300]^{\mathrm{T}}$. The simulation was made over $\mathrm{N}=40$ steps with an interval $[0,2] \mathrm{sec}$. Table 1 shows the mean of the expected values and standard deviations of the neutron density $n(t)$ and the total concentration of precursors $\mathrm{C}_{\mathrm{r}}(\mathrm{t})$ in $\mathrm{t}=2 \mathrm{sec}$ using different seeds with one uncertaint $\Delta \overrightarrow{\mathrm{W}}=\Delta \mathrm{W}_{1}$ where, $\mathrm{C}_{\mathrm{\tau}}(\mathrm{t})=\sum_{\mathrm{i}=\mathrm{i}}^{\mathrm{m}} \mathrm{C}_{\mathrm{i}}(\mathrm{t})$. These results indicate that, for 10 different seeds, each value of the neutron density and concentration of precursors is very similar to the average value, respectively when we use the proposed method $\left(\mathrm{EM}^{*}\right)$, this is using the Euler-Maruyama method with a given uncertainty for Eq.4.
Table 1: Expected values and standard deviations using different seeds for

\begin{tabular}{lcccc}
\hline \multicolumn{5}{c}{ one precursor with one uncertainty } \\
\hline Seeds & $\mathrm{E}(\mathrm{n}(2 \mathrm{ses}))$ & $\sigma(\mathrm{n}(2 \mathrm{sec}))$ & $\mathrm{E}\left(\mathrm{C}_{\tau}(2 \mathrm{sec})\right)$ & $\sigma\left(\mathrm{C}_{\tau}(2 \mathrm{sec})\right)$ \\
100 & 400.1973 & 31.18396 & 299.7546 & 7.929925 \\
200 & 399.7253 & 31.98934 & 299.7701 & 7.966396 \\
300 & 399.2918 & 31.00181 & 299.9346 & 8.056304 \\
400 & 400.4318 & 31.56806 & 299.8427 & 7.794507 \\
500 & 399.8844 & 31.56903 & 300.0402 & 7.840968 \\
600 & 400.3342 & 31.42084 & 299.9319 & 8.028536 \\
700 & 399.4346 & 31.62014 & 299.8181 & 7.955117 \\
800 & 400.2483 & 30.98744 & 299.8795 & 7.883205 \\
900 & 399.0195 & 31.09449 & 299.8779 & 8.017407 \\
1000 & 399.8987 & 31.49116 & 300.1506 & 8.062298 \\
Mean $\left(\mathrm{EM}^{*}\right)$ & 399.8466 & 31.39263 & 299.9000 & 7.953466 \\
\hline \multicolumn{5}{l}{}
\end{tabular}

Table 2: Expected values and standard deviations using different seeds for

\begin{tabular}{lcccc}
\multicolumn{5}{c}{ one precursor with two uncertainties } \\
\hline Seeds & $\mathrm{E}(\mathrm{n}(2 \mathrm{sec}))$ & $\sigma(\mathrm{n}(2 \mathrm{sec}))$ & $\mathrm{E}\left(\mathrm{C}_{\tau}(2 \mathrm{sec})\right)$ & $\sigma\left(\mathrm{C}_{\tau}(2 \mathrm{sec})\right)$ \\
\hline 100 & 400.1021 & 31.57557 & 299.7669 & 10.24146 \\
200 & 399.6746 & 32.40962 & 299.6904 & 10.34983 \\
300 & 399.2808 & 31.43599 & 299.8284 & 10.32776 \\
400 & 400.3723 & 32.00041 & 299.8863 & 10.14301 \\
500 & 399.9058 & 31.96058 & 300.0430 & 10.13379 \\
600 & 400.3183 & 31.83929 & 299.9589 & 10.32397 \\
700 & 399.3583 & 32.01649 & 299.7299 & 10.25591 \\
800 & 400.1835 & 31.37446 & 299.9067 & 10.13157 \\
900 & 398.9828 & 31.50246 & 299.7565 & 10.30098 \\
1000 & 399.9648 & 32.03126 & 300.1290 & 10.47774 \\
Mean $\left(\mathrm{EM}^{* *}\right)$ & 399.8143 & 31.81461 & 299.8696 & 10.26860 \\
\hline
\end{tabular}

Table 3: Comparison of methods in a problem of one precursor

\begin{tabular}{lcccc}
\hline Methods & $\mathrm{E}(\mathrm{n}(2 \mathrm{sec}))$ & $\sigma(\mathrm{n}(2 \mathrm{sec}))$ & $\mathrm{E}\left(\mathrm{C}_{\tau}(2 \mathrm{sec})\right)$ & $\sigma\left(\mathrm{C}_{\tau}(2 \mathrm{sec})\right)$ \\
\hline Monte Carlo & 400.0300 & 27.31100 & 300.0000 & 7.806300 \\
PCA & 395.3200 & 29.41100 & 300.6700 & 8.356400 \\
EM (Saha) & 412.2300 & 34.39100 & 315.9600 & 8.265600 \\
Taylor 1.5 & 412.1000 & 34.51900 & 315.9300 & 8.315800 \\
AEM & 396.2800 & 31.21200 & 300.4200 & 7.957600 \\
ESM & 396.6200 & 0.919900 & 300.3900 & 0.001600 \\
DDDM & 402.1300 & 28.93000 & 305.8400 & 7.924900 \\
EM $^{* *}$ & 399.8143 & 31.81461 & 299.8696 & 10.26860 \\
EM $^{*}$ & 399.8466 & 31.39263 & 299.9000 & 7.953466 \\
RK O(h $\left.^{4}\right)$ & 400.0000 & - & 300.0000 & - \\
\hline
\end{tabular}

Considering that there are two uncertainties $\Delta \mathrm{W}_{1}$ and $\Delta \mathrm{W}_{2}$ associated with each one of the variables we want to know in this case $n(t)$ and $\mathrm{C}_{\mathrm{r}}(\mathrm{t})$. Table 2 shows the mean of expected values and standard deviations of the neutron density $\mathrm{n}(\mathrm{t})$ and the total concentration of precursors $\mathrm{C}_{\mathrm{r}}(\mathrm{t})$ in $\mathrm{t}=2 \mathrm{sec}$. The results shown in Table 1 and 2 indicate that there is a slight change in the expected values but a significant change in the standard deviation for the concentration of precursors which seems reasonable, since, we have considered one uncertainty more than in the first case, represented, by $\left(\mathrm{EM}^{*}\right)$ and $\left(\mathrm{EM}^{* *}\right)$, in this second case the Euler-Maruyama method with two uncertainties given by Eq. 5 is used.

Table 3 shows the comparison of the previous results with other works reported in literature and their validation with the deterministic model. First, we can see that the current proposal has a mean value for the neutron densities and for the very approximate concentration of precursors when we compare it with the deterministic 
Table 4: Expected values and standard deviations for $\rho=0.003$ (or $300 \mathrm{pcm}$ ) using different seeds for one uncertainty

\begin{tabular}{llccc}
\hline Seeds & $\mathrm{E}(\mathrm{n}(0.1 \mathrm{sec}))$ & $\sigma(\mathrm{n}(0.1 \mathrm{sec}))$ & $\mathrm{E}\left(\mathrm{C}_{\mathrm{r}}(0.1 \mathrm{sec})\right)$ & $\sigma\left(\mathrm{C}_{\mathrm{r}}(0.1 \mathrm{sec})\right)$ \\
\hline 100 & 179.3362 & 217.3994 & 448867.8 & 1989.857 \\
200 & 179.0589 & 219.5875 & 448847.9 & 1987.940 \\
300 & 177.1956 & 215.0377 & 448875.7 & 2042.874 \\
400 & 179.1409 & 217.5602 & 448877.8 & 1996.082 \\
500 & 171.9135 & 215.7338 & 448918.2 & 1960.309 \\
600 & 178.4538 & 220.5902 & 448882.3 & 2000.672 \\
700 & 177.1948 & 216.1799 & 448864.9 & 2003.152 \\
800 & 179.5957 & 215.6196 & 448915.2 & 2002.388 \\
900 & 178.7917 & 216.6938 & 448848.5 & 2021.231 \\
1000 & 181.7231 & 217.0913 & 448932.4 & 2039.020 \\
Mean $\left(\mathrm{EM}^{*}\right)$ & 178.2404 & 217.1493 & 448883.9 & 2004.353 \\
\hline
\end{tabular}

value when the Runge-Kutta method is used. In addition, the values obtained in the current proposal, especially when one uncertainty is used are very much in agreement when compared with the results obtained using the Monte Carlo and PCA methods (Hayes and Allen, 2005) and with the AEM method (Nahla and Edress, 2016a).

The second and third experiment consist of the insertion of two reactivity constants for a prompt subcritical insertion $\rho=0.003$ (or equivalently $300 \mathrm{pcm}$, parts per hundred thousand) and for a prompt critical insertion $\rho=0.007$ (or equivalently $700 \mathrm{pcm}$ ), respectively in a problem of six groups of precursors $(m=6)$ for a nuclear reactor with the following parameters: decay constants $\lambda_{i}=[127,317,1150,3110,14000$, $38700] \times 10^{-4} \mathrm{sec}^{-1}$ fractions of delayed neutrons $\beta_{\mathrm{i}}=[266$, $1491,1316,2849,896,182] \times 10^{-6}$, total fraction of delayed neutrons, $\beta=0.007$, fission neutrons $v=2.5$, generation time $\wedge=2 \times 10^{-5} \mathrm{sec}$ and an external source $\mathrm{q}=0 \mathrm{sec}^{-1}$. Both computational experiments were carried out over $\mathrm{N}=40$ steps at intervals of $[0,0.1]$ and $[0,0.001] \mathrm{sec}$ with the initial condition $\vec{P}_{0}=\left[1, \beta_{1} / \lambda_{1} \Lambda, \beta_{2} / \lambda_{2} \Lambda, \cdots, \beta_{6} / \lambda_{6} \Lambda\right]^{\mathrm{T}}$.

Table 4 shows the mean values expected and standard deviations of the neutron density $\mathrm{n}(\mathrm{t})$ and the total concentration of precursors $\mathrm{C}_{\mathrm{t}}(\mathrm{t})$ for a prompt subcritical insertion $\rho=0.003$ (or equivalently $300 \mathrm{pcm}$ ) in $\mathrm{t}=0.1 \mathrm{sec}$, using different seeds with one uncertainty $\Delta \overrightarrow{\mathrm{W}}=\Delta \mathrm{W}_{1}$. Again the results using 10 different seeds, each value with the neuron densities and concentration of precursors are very similar to the average value.

Table 5 shows the mean of the expected values and standard deviations of the neutron density $n(t)$ and the total concentration of precursors $\mathrm{C}_{\mathrm{r}}(\mathrm{t})$ for the same case as Table 4 with the difference that seven uncertainties $\left(\Delta \overrightarrow{\mathrm{W}}=\Delta \mathrm{W}_{\mathrm{i}}, \mathrm{i}=1, \cdots, 7\right)$ are used. These results are approximately the same for the neutron density and concentration of precursors when compared with their average value when using 10 different seeds.

Table 6 compares the previous results with other works reported in literature and the deterministic point kinetic model. It can be observed that the results obtained
Table 5: Expected values and standard deviations for $\rho=0.003$ (or $300 \mathrm{pcm})$ using different seeds with seven uncertainties

\begin{tabular}{lcccc}
\hline Seeds & $E(n(0.1 \mathrm{sec})$ & $\sigma(\mathrm{n}(0.1 \mathrm{sec}))$ & $\mathrm{E}\left(\mathrm{C}_{r}(0.1 \mathrm{sec})\right)$ & $\sigma\left(\mathrm{C}_{r}(0.1 \mathrm{sec})\right)$ \\
\hline 100 & 179.0575 & 217.8088 & 448867.8 & 2174.250 \\
200 & 179.3576 & 219.8941 & 448845.9 & 2179.808 \\
300 & 177.5753 & 216.1228 & 448876.6 & 2241.775 \\
400 & 179.6542 & 218.4496 & 448875.3 & 2186.352 \\
500 & 171.9785 & 216.7798 & 448922.3 & 2144.035 \\
600 & 178.5670 & 221.5763 & 448880.4 & 2188.824 \\
700 & 176.9575 & 216.8416 & 448863.3 & 2197.016 \\
800 & 179.4934 & 216.8765 & 448916.7 & 2186.658 \\
900 & 178.9073 & 217.7606 & 448853.3 & 2217.282 \\
1000 & 181.8915 & 218.4109 & 448940.5 & 2235.841 \\
Mean $\left(\mathrm{EM}^{* *}\right)$ & 178.3439 & 218.0521 & 448884.1 & 2195.184 \\
\hline
\end{tabular}

Table 6: Comparison of methods for $\rho=0.003$ (or $300 \mathrm{pcm}$ ) in a problem of six precursors

\begin{tabular}{lrrrc}
\hline Methods & $\mathrm{E}(\mathrm{n}(0.1))$ & $\sigma(\mathrm{n}(0.1))$ & $\mathrm{E}\left(\mathrm{C}_{\tau}(0.1)\right)$ & $\sigma\left(\mathrm{C}_{\tau}(0.1)\right)$ \\
\hline Monte Carlo & 183.0400 & 168.7900 & 447800.0 & 1495.700 \\
PCA & 186.1600 & 164.1600 & 449100.0 & 1917.200 \\
EM (Saha) & 208.6000 & 255.9500 & 449800.0 & 1233.380 \\
Taylor 1.5 & 199.4000 & 168.5400 & 449700.0 & 1218.800 \\
AEM & 186.3000 & 164.1400 & 449000.0 & 1911.900 \\
ESM & 179.9300 & 10.55500 & 448900.0 & 94.75000 \\
DDDM & 187.0500 & 167.8300 & 448800.0 & 1475.600 \\
EM $^{*}$ & 178.3439 & 218.0521 & 448884.1 & 2195.184 \\
EM $^{*}$ & 178.2404 & 217.1493 & 448883.9 & 2004.353 \\
RK O(h $\left.^{4}\right)$ & 179.9500 & - & 448878.0 & - \\
\hline
\end{tabular}

Table 7: Expected values and standard deviations for $\rho=0.003$ (or $700 \mathrm{pcm})$ using different seeds with one uncertainty

\begin{tabular}{lcccc}
\hline & $E(n$ & $\sigma(n$ & $E\left(C_{\tau}\right.$ & $\sigma\left(C_{\tau}\right.$ \\
Seeds & $(0.001 \mathrm{sec}))$ & $(0.001 \mathrm{sec}))$ & $(0.001 \mathrm{sec}))$ & $(0.001 \mathrm{sec}))$ \\
\hline 100 & 134.2827 & 92.56374 & 446360.5 & 18.79142 \\
200 & 134.0279 & 93.27359 & 446359.9 & 18.77698 \\
300 & 134.1766 & 92.73754 & 446360.3 & 18.62391 \\
400 & 135.6616 & 94.29252 & 446360.4 & 18.81022 \\
500 & 136.6873 & 92.72723 & 446360.6 & 18.62025 \\
600 & 134.4107 & 94.01819 & 446360.1 & 18.90852 \\
700 & 134.3027 & 92.79483 & 446360.2 & 18.52236 \\
800 & 135.9779 & 93.44768 & 446360.5 & 18.66368 \\
900 & 133.0583 & 92.33267 & 446359.8 & 18.66976 \\
1000 & 137.6626 & 93.66755 & 446360.9 & 18.91518 \\
Mean $\left(\mathrm{EM}^{*}\right)$ & 135.0248 & 93.18555 & 446360.3 & 18.73023 \\
\hline
\end{tabular}

using the proposed method with one and seven uncertainties $\left(\mathrm{EM}^{*}\right.$ and $\left.\mathrm{EM}^{* *}\right)$ when compared with an expected value in the deterministic case and is very approximate to the Runge-Kutta methods and the recently published ESM method (Nahla and Edress, 2016b). In the standard deviation, it is similar to the PCA method (Hayes and Allen, 2005). However, there is a difference in the results when compared with the Euler-Maruyama method (Ray, 2012).

For a prompt critical insertion $\rho=0.007$ (or equivalently $700 \mathrm{pcm}$ ), Table 7 shows the mean of the expected values and standard deviations of the neutron density $\mathrm{n}(\mathrm{t})$ and the total concentration of precursors $\mathrm{C}_{\mathrm{r}}(\mathrm{t})$ in $\mathrm{t}=0.001 \mathrm{sec}$, using different seeds with one uncertainty $\Delta \overrightarrow{\mathrm{W}}=\Delta \mathrm{W}_{1}$. The results are very similar and there is practically no difference in each calculation carried out with regard to the expected values and standard deviations. 


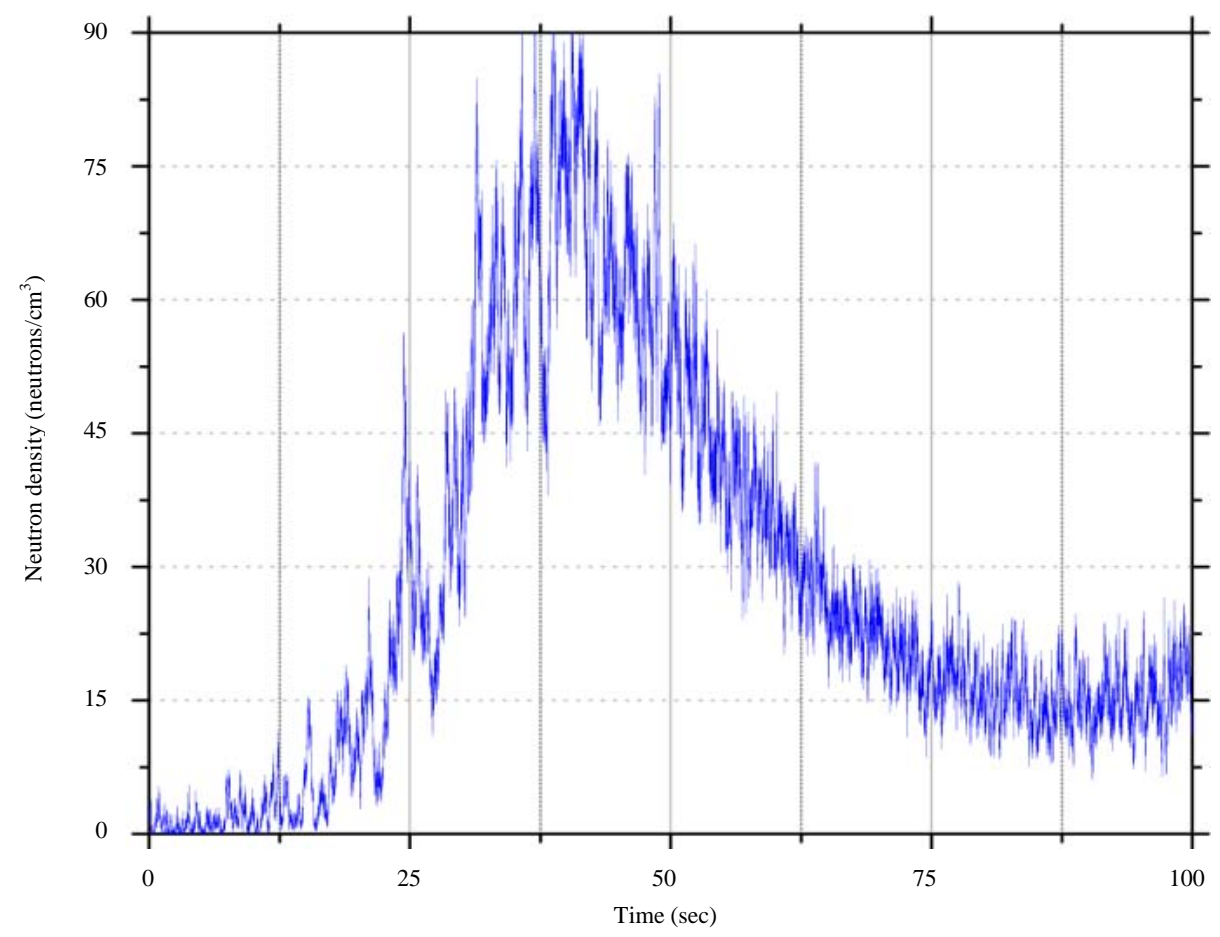

Fig. 1: Density of neutrons for a sinusoidal reactivity through one period

Table 8: Expected values and standard deviations for $\rho=0.003$ (or $700 \mathrm{pcm}$ ) using different seeds with seven uncertainties

\begin{tabular}{lcccc}
\hline \multicolumn{5}{c}{$700 \mathrm{pcm})$ Using different seeds with seven uncertainties } \\
Seeds & $\begin{array}{c}\mathrm{E}(\mathrm{n} \\
(0.001 \mathrm{sec}))\end{array}$ & $\begin{array}{c}\sigma(\mathrm{n} \\
(0.001 \mathrm{sec}))\end{array}$ & $\begin{array}{c}\mathrm{E}\left(\mathrm{C}_{\tau}\right. \\
(0.001 \mathrm{sec}))\end{array}$ & $\begin{array}{c}\sigma\left(\mathrm{C}_{\tau}\right. \\
(0.001 \mathrm{sec}))\end{array}$ \\
\hline 100 & 134.3062 & 92.62652 & 446360.4 & 29.09122 \\
200 & 134.0041 & 93.54258 & 446359.8 & 29.27451 \\
300 & 134.2316 & 93.10647 & 446360.2 & 28.98068 \\
400 & 135.7164 & 94.47060 & 446360.6 & 29.35535 \\
500 & 136.6362 & 92.77626 & 446360.7 & 28.89884 \\
600 & 134.3586 & 94.10905 & 446360.0 & 29.54918 \\
700 & 134.2543 & 93.02594 & 446360.1 & 28.91319 \\
800 & 135.9165 & 93.53007 & 446360.6 & 29.02346 \\
900 & 133.0774 & 92.46126 & 446359.6 & 28.93699 \\
1000 & 137.6863 & 93.81167 & 446361.2 & 29.54030 \\
Mean $\left(\mathrm{EM}^{* *}\right)$ & 135.0188 & 93.34604 & 446360.3 & 29.15637 \\
\hline
\end{tabular}

Table 8 shows the mean of expected results and standard deviations of the neutron density $n(t)$ and the total concentration of precursors $\mathrm{C}_{\mathrm{r}}(\mathrm{t})$ for the same case as Table 7 except that seven uncertainties $\Delta \overrightarrow{\mathrm{W}}=\Delta \mathrm{W}_{\mathrm{i}}, \mathrm{i}=1, \cdots, 7$ are used associated with each of the variables that we want to know in this case $(\mathrm{t})$ and $\mathrm{C}_{\mathrm{r}}(\mathrm{t})$ (Fig. 1-4).

The results obtained for a prompt critical insertion $\rho=0.007$ (or equivalently $700 \mathrm{pcm}$ ) are presented in Table 9 and compare the results of other methods reported in literature, together with their validation with the deterministic model. From these results we can see that the proposed model when an uncertainty $\left(\mathrm{EM}^{*}\right)$ is used or seven uncertainties, $\left(\mathrm{EM}^{* *}\right)$ in the case of the expected values are very precise when compared with the
Table 9: Comparison of methods for $\rho=0.003$ (or $700 \mathrm{pcm}$ ) in a problem of six precursors

\begin{tabular}{lcccc}
\hline \multicolumn{4}{c}{ of six precursors } & \multicolumn{3}{c}{$\begin{array}{l}\sigma(\mathrm{n} \\
\text { Method }\end{array}$} & $\begin{array}{c}\mathrm{E}\left(\mathrm{n}\left(\mathrm{C}_{\tau}\right.\right. \\
(0.001 \mathrm{sec}))\end{array}$ & $\begin{array}{c}\sigma\left(\mathrm{C}_{\tau}\right. \\
(0.001 \mathrm{sec}))\end{array}$ & $(0.001 \mathrm{sec}))$ & $(0.001 \mathrm{sec}))$ \\
\hline Monte Carlo & 135.6700 & 93.37600 & 446400.0 & 16.22600 \\
PCA & 134.5500 & 91.24200 & 446400.0 & 19.44400 \\
EM (Saha) & 139.5680 & 92.04200 & 446300.0 & 6.071000 \\
Taylor 1.5 & 139.5700 & 92.04700 & 446300.0 & 18.33700 \\
AEM & 134.5400 & 91.23400 & 446400.0 & 19.23500 \\
ESM & 134.9600 & 6.852000 & 446400.0 & 2.529000 \\
DDDM & 135.8600 & 93.21000 & 446300.0 & 17.84500 \\
EM & 135.0188 & 93.34604 & 446360.3 & 29.15637 \\
EM $^{*}$ & 135.0248 & 93.18555 & 446360.3 & 18.73023 \\
RK O(h $\left.^{4}\right)$ & 135.0000 & - & 446360.0 & - \\
\hline
\end{tabular}

methods in literature and their exactness can be proven with the Runge-Kutta method. For the standard deviation value for the nuclear density we can say that all methods are very similar also for the standard deviation in the density of precursors the methods presented in the current proposal $\left(\mathrm{EM}^{*}\right)$ and PCA (Hayes and Allen, 2005) practically coincide.

The results given in Table 4, 5, 7, 8, indicate improvements in the approximations by using only one uncertainty. On the other hand, according to Table 6 and 9 , it can be seen that the present proposal is in line with the values reported by other methods (Hayes and Allen, 2005; Nahla and Edress, 2016a, b; Da Silva et al., 2016) while in another publication (Ray, 2012) in which the same method is used there are significant differences. 


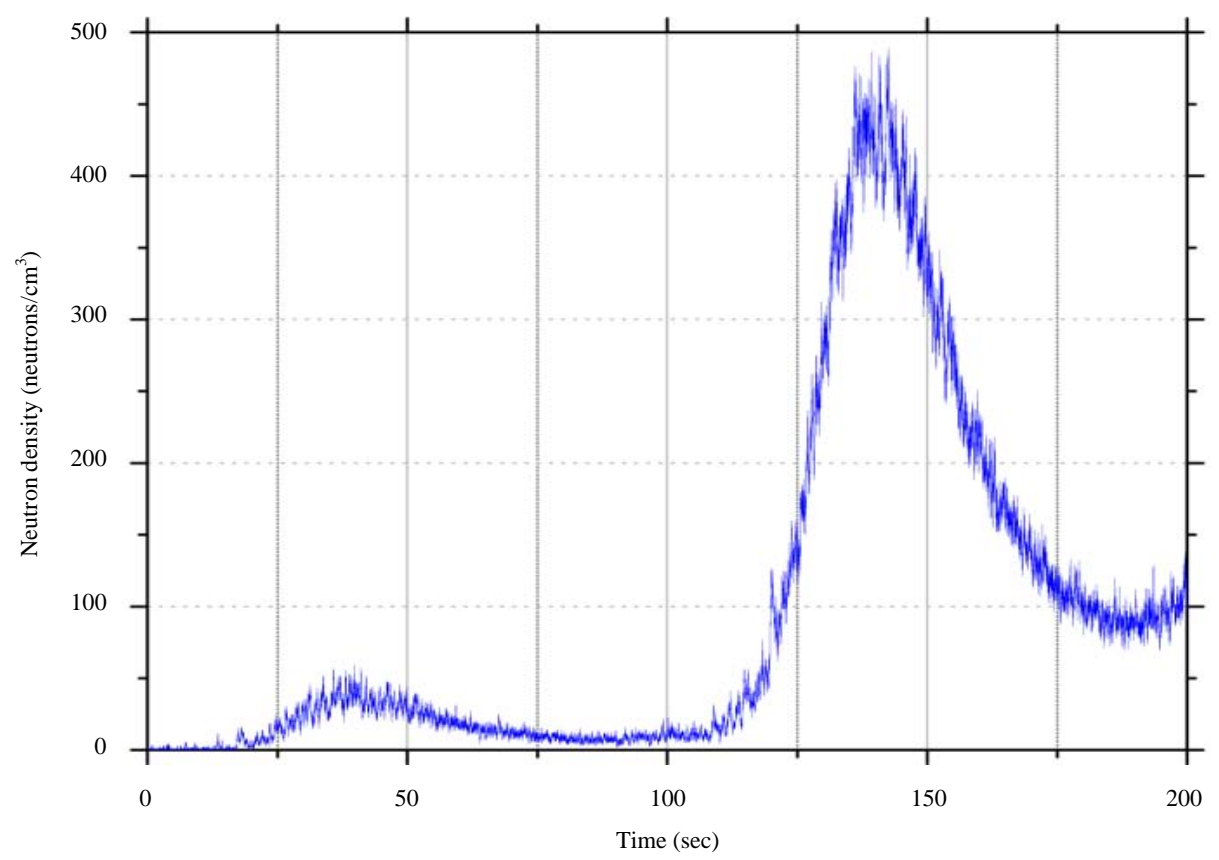

Fig. 2: Density of neutrons for a sinusoidal reactivity through two periods

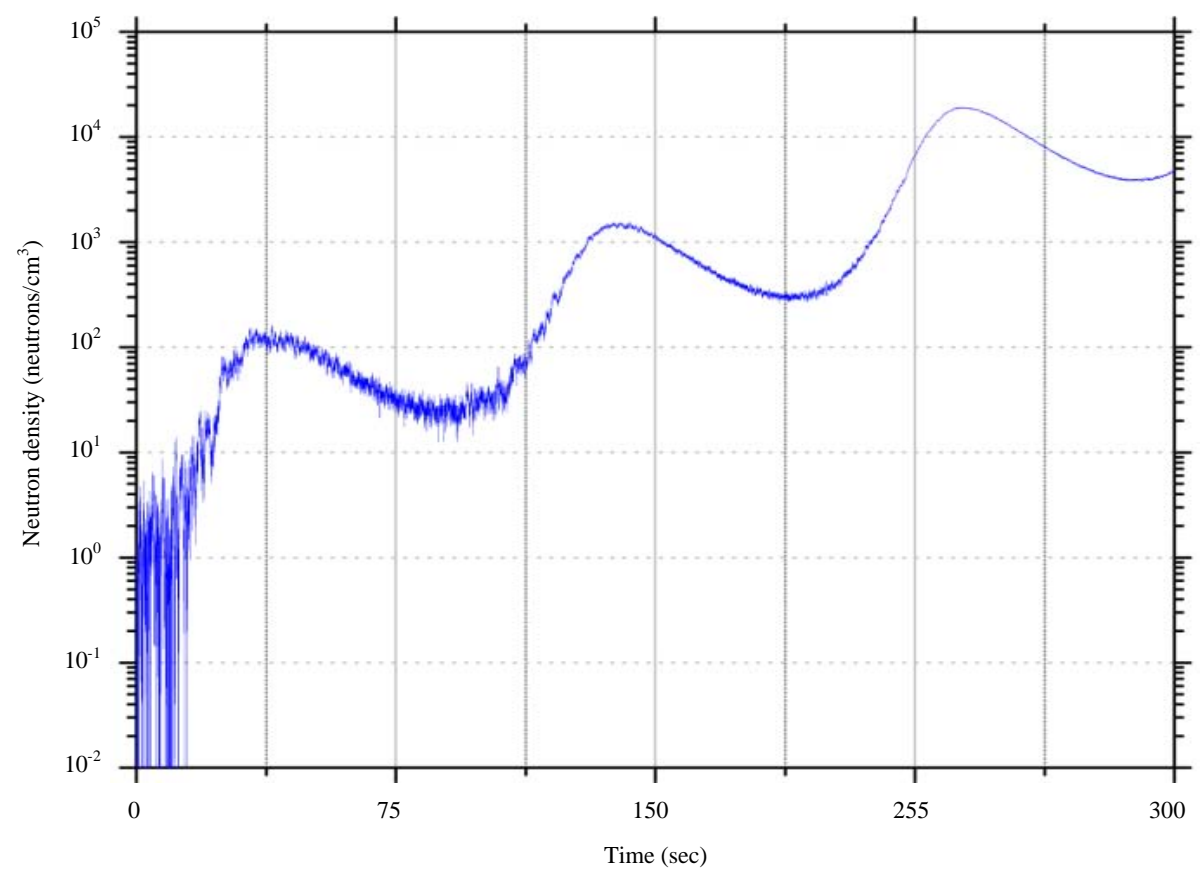

Fig. 3: Density of neutrons for a sinusoidal reactivity through three periods

Finally, the last computational experiments shown (Ray, 2013) consist of a sinusoidal reactivity $\rho(t) \rho_{0} \operatorname{Sin}$ $(\pi \mathrm{t} / \mathrm{T})$ in a problem of one precursor $(\mathrm{m}=1)$. The parameters used for this simulation consist of an initial reactivity $\rho_{0}=0.005333$ (or equivalently $533.3 \mathrm{pcm}$ ), decay constant $\lambda_{1}=0.077 \mathrm{sec}^{-1}$, fraction of neutron precursor $\beta_{1}$ $=0.0079$, time of neutron generation $\Lambda=10^{-3} \mathrm{sec}$, external neutron source $\mathrm{q}=0 \mathrm{sec}^{-1}$, an average period $\mathrm{T}=50$ sec and initial condition $\vec{P}_{0}=,\left[1, \beta_{1} / \lambda_{1} \Lambda\right]^{\mathrm{T}}$. The results obtained are shown in Fig. 1-4 show the expected value 


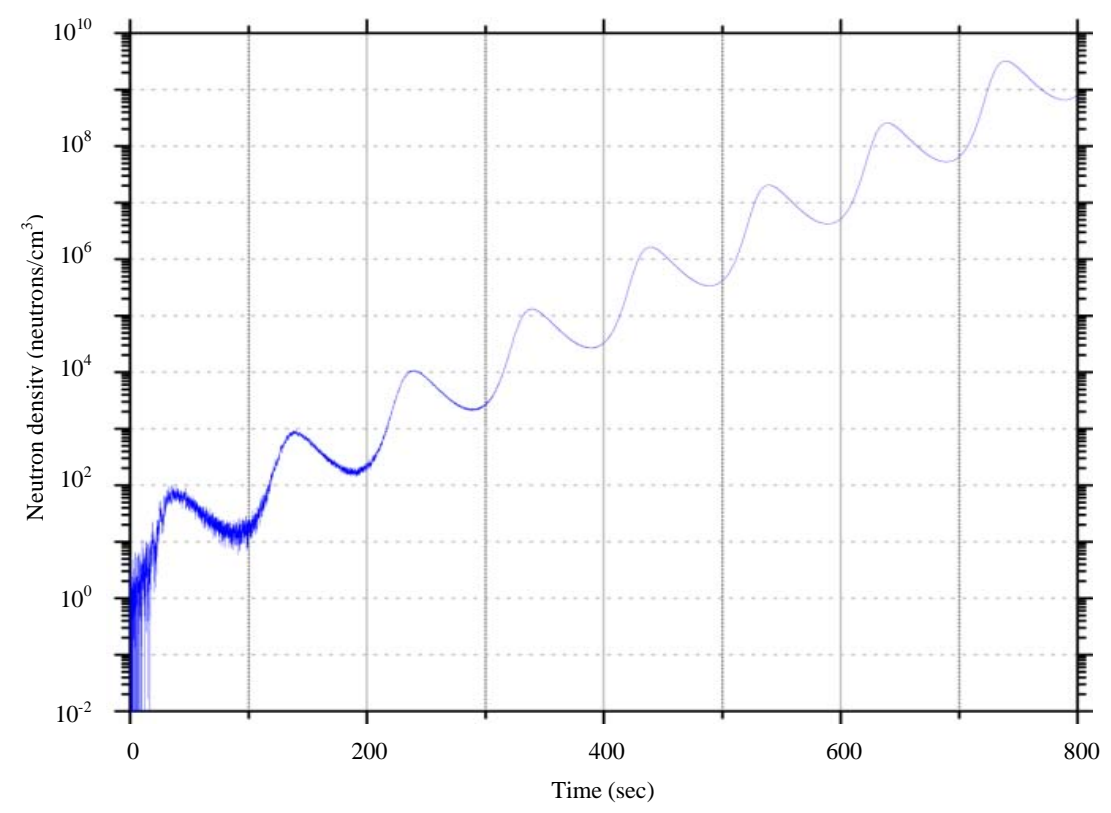

Fig. 4: Density of neutrons for a sinusoidal reactivity through eight periods

of the neutron density for a time interval of $[0.100,0.200$, 0.003 and $0.800 \mathrm{sec}]$, respectively. Comparing the results obtained with the current proposal, under the same parameters we found that there are time intervals considered as one, two, three and eight spikes, respectively. This analysis is supported by recent studies (Nahla and Edress, 2016).

\section{CONCLUSION}

In this study, the stochastic point kinetic equations were numerically solved using the Euler-Maruyama method in the cases in which each random variable $n(t)$ $\mathrm{y}_{\mathrm{i}}(\mathrm{t})$ depends on one or several uncertainties $\Delta \overrightarrow{\mathrm{w}}$. In this way, approximations were obtained of the expected values for the neutron density and delayed neutron precursor concentrations using different seeds with various types of reactivity and initial conditions. The results were compared with other methods reported in literature for which it was possible to demonstrate that this proposal achieved better approximations, confirming that the method, apart from being easy to implement is efficient in the study of stochastic kinetics in a nuclear reactor.

\section{RECOMMENDATIONS}

The researchers consider and recommend making greater efforts in research to propose a new matrix B and/or for the calculation of the square root of the matrix $B$ presented in Eq. 7-5), since, this would enable to establish if it is necessary to use all the uncertainties $\left(\Delta \vec{W}_{i}\right.$, $i=1, \ldots, 7$ ) for each of the unknowns and with this to be able to determine the standard deviation of neutron densities and delayed neutron concentrations. At the same time, more numerical simulations should be carried out with different seeds that provide numbers which would be as random as possible to reconfirm that the results presented in the literature are correct.

The researchers also consider that there is a potential for work to be done by including different considerations in the study and granting different possibilities that can be presented, making the problem a little more general, considering, for example, the inclusion of 8 groups of delayed neutrons and determining how this would affect the average value and standard deviations of neutron densities and concentration. The effect of feedback was not considered in the present research and it would be very interesting to know what the stochastic behavior of the nuclear density would be and if it is also possible to determine the reactivity which is perhaps the most important parameter in a nuclear reactor. Finally, another possibility would be to take the work to stochastic spatial kinetics in 2D and 3D problems, possible in this way we could be able to better describe the real behavior of a nuclear reactor.

\section{ACKNOWLEDGEMENT}

The researcher thank the research seed of Computational Physics, the research group in Applied Physics FIASUR and the academic and financial support of the Universidad Surcolombiana. 


\section{REFERENCES}

Ayyoubzadeh, S.M. and N. Vosoughi, 2014. An alternative stochastic formulation for the point reactor. Ann. Nucl. Energy, 63: 691-695.

Da Silva, M.W., R. Vasques, B.E. Bodmann and M.T. Vilhena, 2016. A nonstiff solution for the stochastic neutron point kinetics equations. Ann. Nucl. Energy, 97: 47-52.

Hayes, J.G. and E.J. Allen, 2005. Stochastic point-kinetics equations in nuclear reactor dynamics. Ann. Nucl. Energy, 32: 572-587.

Kinard, M. and E.J. Allen, 2004. Efficient numerical solution of the point kinetics equations in nuclear reactor dynamics. Ann. Nucl. Energy, 31: 1039-1051.
Nahla, A.A. and A.M. Edress, 2016a. Analytical exponential model for stochastic point kinetics equations via eigenvalues and eigenvectors. Nucl. Sci. Tech., 27: 19-27.

Nahla, A.A. and A.M. Edress, 2016b. Efficient stochastic model for the point kinetics equations. Stochastic Anal. Appl., 34: 598-609.

Ray, S.S. and A. Patra, 2013. Numerical solution for stochastic point-kinetics equations with sinusoidal reactivity in dynamical system of nuclear reactor. Intl J. Nucl. Energy Sci. Technol., 7: 231-242.

Ray, S.S., 2012. Numerical simulation of stochastic point kinetic equation in the dynamical system of nuclear reactor. Ann. Nucl. Energy, 49: 154-159.

Stacey, W.M., 2007. Nuclear Reactor Physics. 2nd Edn., Wiley-Vch, Weinheim, Germany, USA., ISBN: 9783527406791, Pages: 736. 